\title{
(- OPEN ACCESS \\ Epidemiology, management and outcome of ultrashort bowel syndrome in infancy
}

\author{
Akshay Batra, ${ }^{1}$ Simon Charlie Keys, ${ }^{2}$ Mark John Johnson, ${ }^{3}$ Robert A Wheeler, ${ }^{2}$ \\ Robert Mark Beattie ${ }^{1}$
}

'Department of Paediatric

Gastroenterology, Southampton Children's Hospital,

Southampton, UK

${ }^{2}$ Wessex Regional Pediatric

Surgery, Southampton Children's Hospital, Southampton, UK ${ }^{3}$ Department of Neonatal Medicine, Southampton Children's Hospital,

Southampton, UK

\section{Correspondence to}

Professor Robert Mark Beattie, Department of Paediatric Gastroenterology, Southampton Children's Hospital, University Hospital Southampton, Tremona road, Southampton, S016 6YD, UK; rm.beattie@btinternet.com

Received 27 March 2017

Revised 11 July 2017

Accepted 13 July 2017

Published Online First

2 September 2017

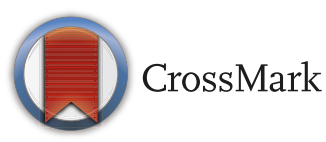

To cite: Batra A, Keys SC Johnson MJ, et al. Arch Dis Child Fetal Neonatal Ed 2017:102:F551-F556.

\section{ABSTRACT}

Ultrashort bowel syndrome (USBS) is a group of heterogeneous disorders where the length of small bowel is less than $10 \mathrm{~cm}$ or $10 \%$ of expected for the age. It is caused by massive loss of the gut which in the neonatal period can be a result of vanishing gastroschisis or surgical resection following midgut volvulus, jejunoileal atresia and/or extensive necrotising enterocolitis. The exact prevalence of USBS is not known although there is a clear trend towards increasing numbers because of increased incidence and improved survival. Long-term parenteral nutrition (PN) is the mainstay of treatment and is best delivered by a multidisciplinary intestinal rehabilitation team. Promoting adaptation is vital to improving long-term survival and can be achieved by optimising feeds, reducing intestinal failure liver disease and catheter-related bloodstream infections. Surgical techniques that can promote enteral tolerance and hence improve outcome include establishing intestinal continuity and bowel lengthening procedures. The outcome for USBS is similar to patients with intestinal failure due to other causes and only a small proportion of children who develop irreversible complications of PN and will need intestinal transplantation. In this review, we will summarise the available evidence focusing particularly on the epidemiology, management strategies and outcome.

\section{INTRODUCTION}

Short bowel syndrome (SBS) is a reduction in functioning bowel length. It is the the most common cause of intestinal failure in children and is the underlying cause in nearly half of the patients on home parenteral nutrition (HPN). ${ }^{1}{ }^{2}$ Its severity has historically been defined in terms of length of residual bowel, measured from the ligament of Treitz. On the basis of length, a subgroup of ultrashort bowel syndrome (USBS) is defined as bowel length less than $10 \mathrm{~cm}$ or less than $10 \%$ of the expected length for age (box). It has historically been considered as a separate subgroup as it was associated with poorer outcomes, including the potential for palliative care in the immediate postnatal period. ${ }^{3}$ These patients previously were referred for early transplant, which was considered the most appropriate long-term treatment option rather than parenteral nutrition (PN). It is important, however, that bowel length, though important, is not the only marker of outcome and bowel quality and function are clearly relevant. The factors influencing outcome include the site of small bowel resected, quality of residual bowel, and presence of the ileocaecal valve, length

\section{Key messages}

- There is an increase in number of children with ultrashort bowel syndrome (USBS) being managed by intestinal rehabilitation services and they form nearly a quarter of children with short bowel syndrome.

- Most of the children and young people will remain on long-term parenteral nutrition.

- There is an improvement in outcome with improved survival and reduced morbidity secondary to complications.

- Only a small proportion of children with USBS will need intestinal transplantation.

of remaining colon and the presence of or potential for intestinal continuity. ${ }^{4}$

The rarity of USBS, combined with a heterogeneous patient group and lack of national and international data, makes accurately defining epidemiology, treatment and outcome a challenging task.

There have been considerable advances in medical management of intestinal failure over the last 10-15 years with coordinated care being provided by multidisciplinary intestinal rehabilitation teams. This has resulted in a significant increase in survival on prolonged $\mathrm{PN}$ with survival probabilities at 2, 5, 10 and 15 years being 97\%, 89\%, 81\% and 72\%, respectively, illustrating that long-term PN now represents a safe and reasonable option. This recognised improvement in long-term outcome and quality of life on prolonged PN has implications for patients with more severe forms of gut loss in the neonatal period and in turn means that robust outcome data for this group would have a direct impact on patient care and influence management decisions during this critical period. ${ }^{5}{ }^{6}$ There have also been significant advances in surgical management with the potential for improving bowel function with non-transplant surgical procedures. As the management of SBS advances and outcome data are becoming more available, it poses the question to the neonatal surgeon as to what they should do when confronted with the patient at laparotomy who has USBS. Furthermore, what should they do with the patient at each end of the spectrum of USBS? Is it appropriate to offer palliation to this patient group?

In this review, we will summarise the available evidence focusing particularly on the epidemiology, management strategies and outcome of children with USBS. 
Box Definition of short and ultrashort bowel syndrome

Short bowel syndrome (SBS) is defined as the loss of length of the small intestine, congenital or following surgical resection, below a minimum required for adequate absorption of enteral nutrients. It cannot be defined only in terms of length of residual bowel as is dependent on the site of bowel resected and quality of bowel.

Ultrashort bowel syndrome is a subgroup of SBS where the length of bowel is less than $10 \mathrm{~cm}$ or less than $10 \%$ of the expected length for age.

\section{Epidemiology}

USBS is most often a result of massive loss of the gut which in the neonatal period can be a result of vanishing gastroschisis or surgical resection following mid-gut volvulus, jejunoileal atresia and extensive necrotising enterocolitis. USBS is rare in older children and if present is usually secondary to bowel ischaemia particularly following complex cardiac surgery.

The problem with defining the incidence of USBS is the hidden mortality of patients with most severe forms who either do not receive active treatment or despite treatment do not survive the neonatal period. This therefore means we can only extrapolate from the number of children on long-term PN who have USBS as their underlying pathology.

Literature specific to paediatric USBS is scarce and the exact incidence is not known, but we presume that the incidence is a proportion of SBS. A large single population-based study from Canada found the incidence of SBS to be 24.5 per 100000 live births with a higher incidence in neonates born before 37 weeks' gestation (353.7/100000 live births). ${ }^{7}$ There are only a few reports in the literature which refer specifically to USBS within a cohort of children with SBS. Sanchez et al described 80 children with SBS seen over a 5-year period in a single centre of whom only five had USBS. ${ }^{7}$ Diamanti et al described a cohort of 38 infants with SBS over 3 years and 11 out of these had USBS. ${ }^{8}$ Burghardt et al studied 91 children with intestinal failure of which 11 had USBS. ${ }^{9}$

\section{Initial management}

It is not always possible to predict preoperatively when the neonatal surgeon is going to be faced with such dramatic operative findings as $<10 \mathrm{~cm}$ viable small bowel. The most likely scenarios are during an operation for a presumed high atresia or during an operation for malrotation and mid-gut volvulus. There may or may not be the presence of ischaemic or necrotic bowel. The decision-making process for the neonatal surgeon in this scenario hinges on the appropriateness of further management of the patient versus palliation. Traditionally, surgeons encountering this situation took it upon themselves to make an intraoperative decision relating to whether treatment with the aim of cure was to be continued. This decision may not need to be faced immediately in the operating theatre by the surgeon acting alone. The surgeon has a role to play in diligently recording the anatomy and resolving immediate technical imperatives; but it is highly desirable that the first approach to the parent should be made jointly by the neonatologist, gastroenterologist and surgeon. The words and tone used to describe the situation, prognosis and options may be determinative of the parents' final decisions. In most cases, it seems unlikely that a clinician will entertain legal severance of the child from his family as a reasonable method of securing their preferred clinical plan, although it is conceded that this presumption may evolve as survival and quality of life for these children improve.

Nonetheless, the decision as to whether active or palliative treatment is appropriate is plainly complex. It is trite law (implying that the legal point is settled and unlikely to change in the foreseeable future) that the child's interests are paramount, and remain at the very least a high priority in most cultures. But the parents will have powerful engrained beliefs and values. The clinicians will have equally powerful (and not necessary similar) views, added to clinical experiences that may be unforgettable and influential. Accordingly, decisions about the best interests of the neonate with USBS can only be forged by accumulating clinical consensus from all practitioners. Only once this preliminary clinical 'view' is established may the parents test it. Once that dialogue commences, the situation will eventually be resolved. In discussion around life-sustaining treatments, it is important to understand that the correct question to ask (at any juncture in the treatment) is whether it is in the child's best interests that PN (which will artificially prolong life) should be continued. If in the clinical circumstances, the answer is that his best interests are not served by future PN, then withdrawal of treatment is lawful; and furthermore, that continuation of the PN (now counter to the best interests) would be unlawful. Posed in this way, the question is equally pertinent before treatment has begun as it is in circumstances when treatment has already been started. It follows that while withdrawal may have different emotional overtones to withholding, there is no legal distinction between them.

At the initial operation, it is crucial to document the length of remaining bowel and distinguish between jejunum and ileum. Some comment on whether the duodenum appears normal or abnormal is helpful as there may be potential for duodenal absorption. The presence of the ileocaecal valve should be documented and the length and quality of remaining colon. General condition of the bowel should be considered including any dilated segments. Assessment of mucosal integrity at resection margins should also be noted. In summary, the initial operation is the best opportunity for the surgeon to accurately document the exact anatomy of the patient to indicate to the multidisciplinary team and family where the patient falls within the spectrum of USBS.

\section{Central venous access}

In a patient with USBS, it is predictable that long-term PN will be required and recurrent central venous infection can increase the risk of liver disease and need for transplantation. ${ }^{10}$ With this in mind, all attempts should be made to use peripherally inserted central venous catheters for as long as possible in the neonatal and early infancy stages, as adopting a central vein preservation approach will increase the likelihood of successful long-term PN. When peripheral access is exhausted, all attempts to insert central venous access should be percutaneous as opposed to open cutdown techniques as it is well established that this is beneficial in preserving veins for repeated cannulation in the long term. ${ }^{11}$

\section{Continued supportive management of USBS}

As USBS is the extreme end of SBS spectrum, the aims of treatment and strategies used are not different from those in children with SBS with some unique problems faced because of a very short bowel length. The principle of management is to promote gut adaptation with the ultimate aim of achieving enteral autonomy while supporting growth and nutrition with PN. 


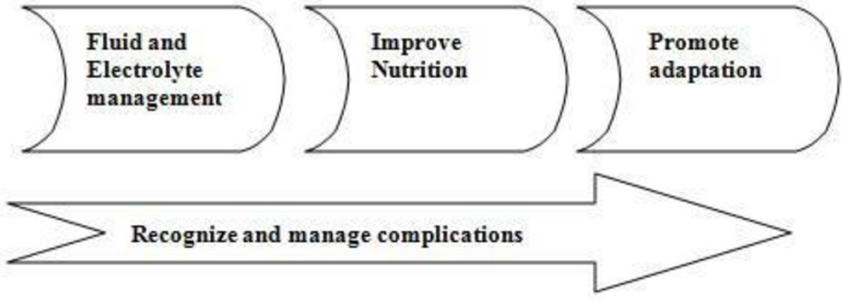

Figure 1 Stages of management of intestinal failure in ultrashort bowel syndrome.

There are three phases of nutritional management in infants with USBS (figure 1). ${ }^{12}$

In the early stages after bowel resection, there are increased losses of fluids and electrolytes which can lead to significant electrolyte imbalance and dehydration. Early restoration of fluid and electrolyte homeostasis is therefore required and necessitates aggressive resuscitation with fluids.

The second phase involves providing macro and micronutrients to promote growth and development. Use of long-term PN, delivered via a multidisciplinary nutritional support team, is the treatment of choice and needs to be targeted towards maintaining a balance between metabolic needs and potential complications. The main aims of nutritional management are to maintain growth and development, promote intestinal adaptation and prevent complications. ${ }^{12}$ A well-balanced energy supply of proteins, carbohydrates and fats is essential to sustain growth. Nutritional requirements can be estimated in most cases based on their corrected age, degree of undernutrition and underlying disease, though it is debatable whether a massive loss of metabolically active bowel tissue would lead to reduced calorie requirements. Approximately $75 \%$ of non-protein calories should be provided as carbohydrate and $25 \%$ as fat. ${ }^{13}$ Amino acids are essential for protein accretion and their requirements can vary depending on the rate of net protein synthesis. The requirements in preterm infants can be as high as $4 \mathrm{~g} / \mathrm{kg} /$ day coming down to around $1 \mathrm{~g} / \mathrm{kg} /$ day after 1 year of age. ${ }^{14}$ Increasing use of lipids with lower omega $6 / 3$ fatty acid ratio and administration of fish oil has shown to reduce risk of intestinal failure liver disease (IFALD). Similar results have been reported with continued use of soya-based lipid but reducing the amount of fat to $1 \mathrm{~g} / \mathrm{kg} / \mathrm{day} .{ }^{15}$

The third phase is promoting gut adaptation. Feeding should be started early in all children irrespective of the length of bowel as it promotes adaptation and reduces risk of complications associated with long-term use of PN. ${ }^{16}$ The amount and type of feed will depend on the total length, quality and type of bowel. Breast milk is the feed of choice as it contains glutamine, growth factors and immunoglobulins which promote adaptation and support the immune system. The high lactose content in breast milk however can limit tolerance due to reduced absorptive surface and disaccharidase activity and artificial formulas may need to be used in a significant proportion. Artificial formulas, when used, should be those which are easily tolerated and promote adaptation. The carbohydrate of choice would be glucose polymer rather than lactose. Even though whole protein feeds are better at promoting intestinal adaptation they are not as well tolerated and hydrolysed protein feeds are better suited for this group. Fat is best delivered as a mixture of long and medium-chain fats to promote adaptation and maximise absorption. ${ }^{12}$ Gut adaptation can be promoted pharmacologically by using trophic hormones. GLP-2 has been reported to improve enteral tolerance and help achieve enteral autonomy in children with various types of IF including USBS. ${ }^{17}$ The restrictions with its use are high cost, reversal of effects on discontinuing treatment and potential risk of malignancy with long-term use. The other promising agent used in adult trials is oral insulin, but its effect needs to be assessed in children with USBS. ${ }^{18}$ Due to short bowel length feeds with high osmolality are generally less well tolerated and can cause osmotic diarrhoea. Wherever possible, the most physiological mode of feeding should be chosen. This ideally should be in form of oral bolus feeding via breast or bottle and progressing on to weaning. In infants who are unable to tolerate oral feeds, nasogastric tube feeding becomes necessary. The most effective feeding strategy to encourage adaptation is likely to involve continuous feeding regimen over 20 hours, which is associated with increased feed tolerance by increasing mucosal contact and increasing transit time within the gut. As tolerance improves, this should be progressed on to bolus feeding through the day with overnight pump feeding. In the early stages, a nasogastric tube is well tolerated but early consideration may be given to insertion of a gastrostomy. This has advantages including ability to give overnight feeds and the ability to decompress the stomach if there is gastric hypersecretion which may help reduce high stoma losses if present.

\section{Central line infections}

Management of catheter-related bloodstream infections (CRBSI) is of paramount importance in reducing mortality and need for transplantation. This is especially true for children with USBS because of potential of lifelong dependence on PN. Units managing children with IF should have established protocols for management of CRBSI. Infections can be prevented by minimising access to lines, using strict aseptic techniques and using bactericidal line locks such as taurolodine or ethanol. ${ }^{19}$

\section{Bacterial overgrowth}

There is a high risk of small bowel bacterial overgrowth (SBBO) because of the increased amount of unabsorbed carbohydrates providing substrate for bacterial growth, intestinal dysmotility and potentially the absence of the ileocaecal valve. The diagnosis can be confirmed by hydrogen/methane breath testing after ingestion of lactulose or glucose. Culture of duodenal juice is helpful to isolate specific pathogens and inform treatment regimens. Early treatment of SBBO helps improve uptake of enteral nutrients, limit damage to the liver and improve intestinal function. ${ }^{20}$ Aggressive management of SBBO includes treatment with antibiotics which are not absorbed from the intestinal mucosa such as gentamycin (intravenous preparation given orally) or rifaximin and using probiotics to promote healthy intestinal microflora.

\section{Intestinal continuity}

The benefits of restoration of intestinal continuity by joining the small bowel remnant to the colon need to be considered. It provides a distinct advantage of increasing enteral tolerance by increasing the absorptive surface and prolonging transit time. It may also allow colonic adaptation to occur which will increase absorption of short-chain fatty acids which can potentially be an additional source of enteral calories. However, the disadvantage of continuity may be high stool output with watery diarrhoea which can be difficult to manage and cause perianal complications. Careful replacement of fluid and electrolytes is essential and some cases with high stool output (stoma output $>30 \mathrm{~mL} /$ $\mathrm{kg},>6$ watery stools/day) may require antidiarrhoeal agents like loperamide or antisecretory agents, for example, proton pump 
inhibitors or clonidine. In children where the colon cannot be in continuity because of high output, the options are to keep the proximal small bowel loop drained with a nasojejunal tube until a definitive management plan has been formed, create a tube enterostomy or create a standard spouted stoma. A tube enterostomy is made by bringing the bowel to the surface of the abdominal wall and creating a small opening big enough to insert a tube the size of a Foley catheter. ${ }^{21}$ The advantage is that bowel length is not lost in creating a spout as in a traditional stoma, and in the event of reversal some length is preserved. With a tube enterostomy, it is also easier to control the output which is likely to be high and watery by spiggoting. This gives the option to 'expansion recycle' should lengthening be considered and also reduces loss of length in subsequent closure. ${ }^{22}$

\section{Role of non-transplant surgery}

The principle is to improve nutrient absorption by increasing food contact time with the intestinal lumen, reducing stasis and bacterial overgrowth. Various autologous gastrointestinal reconstructive procedures have been described and outcomes reported. These include the longitudinal intestinal lengthening and tailoring (or Bianchi) procedure, the serial transverse enteroplasty procedure (STEP) and more recently the spiral intestinal lengthening and tailoring procedure. All of these procedures rely on a dilated segment of small bowel to be present in order to be lengthened although not all patients with USBS will have this. Georgeson et al ${ }^{21}$ described the concept of creating a dilated segment of small bowel using tube enterostomies in the 'controllable expansion recycle' technique which can then be lengthened. Wales et al described five patients with bowel length $<20 \mathrm{~cm}$ who had STEP procedures. ${ }^{23}$ They demonstrated improvement in enteral tolerance in four of five patients, with two achieving enteral autonomy and a further two able to tolerate $>50 \%$ calories enterally. One of five developed significant IFALD and needed liver transplantation. Despite this, it is unlikely that lengthening procedures in USBS would lead to enteral autonomy in view of the small length to start with. Dore et al showed that intestinal adaptation was achieved in 41\% of children with SBS, but a trend towards a poorer outcome was observed in cases with USBS. ${ }^{24}$ Lengthening procedures should therefore be reserved for specially selected cases of USBS. ${ }^{7}$

\section{Indications and timing of intestinal transplantation}

While primary management of IF secondary to USBS is longterm PN, transplantation needs to be considered in cases of PN failure. Failure of PN is defined as development of IFALD, frequent life-threatening episodes of catheter-related sepsis or loss of vascular access. ${ }^{9}$ The incidence of the above complications is reducing, thus reducing the need for transplantation. The indications for paediatric transplantation were developed by an expert consensus and published in 2001 by Kaufman et al. ${ }^{3}$ These included massive resection of bowel resulting in USBS as a primary indication. Burghardt et al undertook a single centre study to assess validity of these criteria and found USBS to be a poor predictor. ${ }^{9}$ Ten of 11 patients in this group survived without needing transplantation. Pironi et al in a recent retrospective survey showed that mortality in children with USBS was lower on home PN (11.1\%) as compared with that after transplant $(16.7 \%),{ }^{25}$ although the cases that went to transplantation are likely to have been a sicker group. The above suggests that transplantation should be the second-line treatment reserved for cases where there is failure of long-term PN. Outcome following intestinal transplant continues to improve and survival post-transplant is $95 \%$ after 1 year, $77 \%$ after 5 years and $46 \%$ at the end of 10 years. ${ }^{26}$ These outcomes are similar to other solid organ transplantation ${ }^{26}$ (table 1).

\section{Long-term outcome}

Children with irreversible intestinal failureare dependent on long-term PN and when that fails need intestinal transplantation. The survival on long-term PN has improved and nearly three quarters of children on long-term PN are expected to be alive at 15 years, ${ }^{5}$ making it the mainstay treatment for children with intestinal failure. The literature on paediatric USBS is scarce and long-term outcomes data in this group from the most recent era of multidisciplinary intestinal failure management are limited. Any recorded outcome data are for children with USBS on long-term PN and do not include children where treatment was withdrawn or those who died in the neonatal period. Even in this small subgroup there is a spectrum of disease, at the worst end of the spectrum is the patient who has lost all intestines in the superior mesenteric artery territory and has no small bowel beyond the second part of the duodenum with only the left side of colon. In contrast, the best end of the spectrum is the patient who has normal duodenum, $10 \mathrm{~cm}$ of small bowel, ileocaecal valve and entire colon. Every variation in between these two ends of the spectrum is possible and some credence must be given to the underlying bowel pathology in terms of potential future function.

Historically, the outcome and survival after bowel resection were linked mainly to the length of residual bowel and this group of patients had especially poor outcome. ${ }^{27}$ Now with the advancement of medical and surgical practices there has been a reduction in mortality, morbidity and need for intestinal transplantation in children with IF especially with USBS. ${ }^{1025}$ A single centre study looked at outcome and need for transplantation in children with USBS. They compared outcomes in 1998-2005 to 2006-2012. This showed that ultrashort bowel was associated with poor outcome in $100 \%$ of the cases in the first group but in only $9 \%(10 / 11)$ in the group from 2006 to 2012.

Mortality in patients with USBS is similar to patients with IF secondary to other causes. In the first year of life it is more likely related to underlying disease; this becomes very important with increasing number of infants with multiple comorbidities being offered active treatment. Beyond this is related to ongoing use of PN and the associated complications. ${ }^{25}$ Pironi et al looked

Table 1 Comparative data of outcomes following different types of solid organ transplants ${ }^{25}$

\begin{tabular}{|c|c|c|c|c|c|c|}
\hline \multirow[b]{2}{*}{ Type of transplant } & \multicolumn{3}{|c|}{ Patient survival (\%) } & \multicolumn{3}{|c|}{ Graft survival (\%) } \\
\hline & 1 year & 5 years & 10 years & 1 year & 5 years & 10 years \\
\hline Renal & 97.4 & 93.3 & 86.6 & 95.1 & $66-78.0$ & 51 \\
\hline Liver & $84-89.8$ & $82-84.8$ & 77 & $84-93$ & $81-88$ & 75 \\
\hline Intestinal & $80-95$ & 77 & 46 & 88 & 74 & 58 \\
\hline
\end{tabular}


Table 2 Outcome in children with ultrashort bowel syndrome

\begin{tabular}{|c|c|c|c|c|c|c|}
\hline & Sanchez et $a l^{7}$ & Diamanti et $a l^{\beta}$ & Pironi et $a l^{25}$ & Burghardt et al ${ }^{p}$ & Wales et $\mathrm{al}^{23}$ & Dore et $a P^{32}$ \\
\hline Number of patients & 5 & 11 & & 11 & 5 & 30 \\
\hline Mortality & 0 & $2(18 \%)$ & $11.5 \%$ & $1(9 \%)$ & 0 & $5(17 \%)$ \\
\hline Transplantation & 1 & 1 & & & 1 & $21(70 \%)$ \\
\hline $\begin{array}{l}\text { Intestinal failure } \\
\text { associated liver disease }\end{array}$ & 0 & 1 & & & 0 & $63 \%$ \\
\hline Enteral autonomy & & $1(9 \%)$ & & & $2(40 \%)$ & 0 \\
\hline
\end{tabular}

at outcome on HPN for both children and adults and included 150 children (total 545) in a large multicentre study. They demonstrated a mortality rate of $11.1 \%$ in patients with USBS which was similar to mortality in all groups (11.5\%) and $75 \%$ of patients with USBS were stable on home PN at 5 years. ${ }^{25}$ Sanchez et al reported outcome in five children with USBS. The children had bowel length of 1-10 cm (median $6 \mathrm{~cm}$ ). This was following surgical resection for various aetiologies including necrotising enterocolitis, gastroschisis, illeal atresia and mid-gut volvulus. Over a median follow-up period of 54 months (43-62), no child died. One had intestinal transplantation at 3 years of age and one was waiting for transplant. Diamanti et al presented data on USBS (bowel length between 3 and $9 \mathrm{~cm}$ ) which included 11 patients of whom nine were alive at the end of follow-up period ranging from 11.5 years. ${ }^{8}$ One patient was able to achieve enteral autonomy and one was able to wean off PN following an intestinal transplant. The rest were increasing their enteral intake receiving between $30 \%$ and $60 \%$ calories via PN.

The prevalence of IFALD is reducing in all patients on longterm PN but there are few studies reporting prevalence of IFALD in various subgroups of IF, making it difficult to define the exact prevalence in this group. In a recent retrospective study on 279 hospitalised children receiving long-term PN, 22\% developed IFALD and $4 \%$ progressed to end-stage liver disease. ${ }^{28}$ This is down from prevalence of approximately 40\%-60\% reported previously in $1998 .^{29}$ In patients with established IFALD, mortality continues to be high and is the main indication for intestinal transplantation in children. ${ }^{30}$ Similar to above data in the case series presented in table 2, IFALD was rare but when present was associated with high morbidity and mortality.

The prevalence of central venous catheter (CVC) infections in this group was similar to that in other patients with IF. Diamanti et al reported an incidence of $<1 / 1000 \mathrm{PN}$ days. ${ }^{8}$ There was no mortality secondary to sepsis in the series presented (table 1). The duration of inpatient care can be variable and ranged from 23 to 104 days per year, was mostly because of CVC infections and constituted only $6 \%-28 \%$ of the total duration on HPN. ${ }^{8}$

It should be highlighted that there is a long-term impact on both patients as well as carers because of the burden of care secondary to providing PN at home. Most patients and carers would have multiple interruptions to sleep at night because of pump alarming, toileting and emptying of stoma/gastrostomy bag. On an average, a child with IF secondary to SBS would be woken up approximately three times per night. There is an added burden of providing enteral tube feeding as boluses or via pumps in most children both in the day and night. ${ }^{31}$

\section{CONCLUSION}

There have been considerable advances in the management of intestinal failure and long-term $\mathrm{PN}$ is now a realistic and safe long-term option in USBS. The incidence of USBS is increasing and forms approximately a quarter of patients with SBS managed in any intestinal rehabilitation programme. This reflects an improvement in the long-term outcome of children with intestinal failure but may also reflect a shift in society and clinicians' attitude towards active management of patients with ever shorter lengths of bowel who previously would have been palliated.

It is part of a continuum of SBS and even within the small subcategory of USBS a spectrum of condition exists. The outcomes in this group are improving and are comparable to other causes of intestinal failure. The emphasis of management is geared towards provision of high-quality care in an intestinal rehabilitation centre and minimising complications.

There is a paucity of data on incidence and long-term outcome in this group and better outcome data of all patients with intestinal failure are essential. This information is required by clinicians who deal with these complex cases and need to give up-to-date accurate information to the families when counselling and planning management which has significant long-term implications.

Contributors $A B$ and RMB conceived the article. All of the authors contributed to the article and approved the final version.

Competing interests None declared.

Provenance and peer review Commissioned; externally peer reviewed.

Open Access This is an Open Access article distributed in accordance with the Creative Commons Attribution Non Commercial (CC BY-NC 4.0) license, which permits others to distribute, remix, adapt, build upon this work non-commercially, and license their derivative works on different terms, provided the original work is properly cited and the use is non-commercial. See: http://creativecommons.org/ licenses/by-nc/4.0/

(C) Article author(s) (or their employer(s) unless otherwise stated in the text of the article) 2017. All rights reserved. No commercial use is permitted unless otherwise expressly granted.

\section{REFERENCES}

1 Guarino A, De Marco G. Italian National Network for Pediatric Intestinal Failure. Natural history of intestinal failure, investigated through a national network-based approach. J Pediatr Gastroenterol Nutr 2003;37:136-41.

2 Squires RH, Duggan C, Teitelbaum DH, et al. Natural history of pediatric intestinal failure: initial report from the Pediatric Intestinal Failure Consortium. J Pediatr 2012;161:723-8.

3 Kaufman SS, Atkinson JB, Bianchi A, et al. Indications for pediatric intestinal transplantation: a position paper of the American Society of Transplantation. Pediatr Transplant 2001;5:80-7.

4 Quirós-Tejeira RE, Ament ME, Reyen L, et al. Long-term parenteral nutritional support and intestinal adaptation in children with short bowel syndrome: a 25-year experience. J Pediatr 2004;145:157-63.

5 Colomb V, Dabbas-Tyan M, Taupin P, et al. Long-term outcome of children receiving home parenteral nutrition: a 20-year single-center experience in 302 patients. J Pediatr Gastroenterol Nutr 2007;44:347-53.

6 Mutanen A, Kosola S, Merras-Salmio L, et al. Long-term health-related quality of life of patients with pediatric onset intestinal failure. J Pediatr Surg 2015;50:1854-8

7 Wales PW, de Silva N, Kim J, et al. Neonatal short bowel syndrome: population-based estimates of incidence and mortality rates. J Pediatr Surg 2004;39:690-5.

8 Diamanti A, Conforti A, Panetta F, et al. Long-term outcome of home parenteral nutrition in patients with ultra-short bowel syndrome. J Pediatr Gastroenterol Nutr 2014;58:438-42

9 Burghardt KM, Wales PW, de Silva N, et al. Pediatric intestinal transplant listing criteria-a call for a change in the new era of intestinal failure outcomes. Am J Transplant 2015;15:1674-81. 
10 Colomb V, Fabeiro M, Dabbas M, et al. Central venous catheter-related infections in children on long-term home parenteral nutrition: incidence and risk factors. Clin Nutr 2000;19:355-9.

11 Male C, Julian JA, Massicotte P, et al. Significant association with location of central venous line placement and risk of venous thrombosis in children. Thromb Haemost 2005;94:516-21.

12 Batra A, Beattie RM. Management of short bowel syndrome in infancy. Early Hum Dev 2013:89:899-904

13 Koletzko B, Goulet O, Hunt J, et al. 1. Guidelines on Paediatric Parenteral Nutrition of the European Society of Paediatric Gastroenterology, Hepatology and Nutrition (ESPGHAN) and the European Society for Clinical Nutrition and Metabolism (ESPEN), Supported by the European Society of Paediatric Research (ESPR). J Pediatr Gastroenterol Nutr 2005;41(Suppl 2):S1-4.

14 de Meijer VE, Gura KM, Le HD, et al. Fish oil-based lipid emulsions prevent and reverse parenteral nutrition-associated liver disease: the Boston experience. JPEN J Parenter Enteral Nutr 2009;33:541-7.

15 Sanchez SE, Braun LP, Mercer LD, et al. The effect of lipid restriction on the prevention of parenteral nutrition-associated cholestasis in surgical infants. J Pediatr Surg 2013:48:573-8.

16 Gosselin KB, Duggan C. Enteral nutrition in the management of pediatric intestinal failure. J Pediatr 2014;165:1085-90.

17 Carter BA, Hill S, Horslen S, et al. SUN-LB011: Successful intestinal adaptation with teduglutide in children with short bowel syndrome (SBS). Clinical Nutrition 2015;34:S239.

18 Shamir R, Kolacek S, Koletzko S, et al. Oral insulin supplementation in paediatric short bowel disease: a pilot observational study. J Pediatr Gastroenterol Nutr 2009;49:108-11.

19 Tan M, Lau J, Guglielmo BJ. Ethanol locks in the prevention and treatment of catheterrelated bloodstream infections. Ann Pharmacother 2014;48:607-15.

20 Engstrand Lilja $\mathrm{H}$, Wefer $\mathrm{H}$, Nyström N, et al. Intestinal dysbiosis in children with short bowel syndrome is associated with impaired outcome. Microbiome 2015;3:18.
21 Georgeson K, Halpin D, Figueroa R, et al. Sequential intestinal lengthening procedures for refractory short bowel syndrome. J Pediatr Surg 1994;29:316-21.

22 Bianchi A. From the cradle to enteral autonomy: the role of autologous gastrointestinal reconstruction. Gastroenterology 2006;130:S138-46.

23 Wales PW, Jancelewicz T, Romao RL, et al. Delayed primary serial transverse enteroplasty as a novel management strategy for infants with congenital ultra-short bowel syndrome. J Pediatr Surg 2013;48:993-9.

24 Dore M, Junco PT, Andres AM, et al. Surgical Rehabilitation Techniques in Children with Poor Prognosis Short Bowel Syndrome. Eur J Pediatr Surg 2016;26:112-6.

25 Pironi L, Joly F, Forbes A, et al. Home Artificial Nutrition \& Chronic Intestinal FailureWorking Group of the European Society for Clinical Nutrition and Metabolism (ESPEN). Long-term follow-up of patients on home parenteral nutrition in Europe:implications for intestinal transplantation. Gut 2011;60:17-25.

26 Kim JJ, Marks SD. Long-term outcomes of children after solid organ transplantation. Clinics 2014;69(Suppl 1):28-38.

27 Quirós-Tejeira RE, Ament ME, Reyen L, et al. Long-term parenteral nutritional support and intestinal adaptation in children with short bowel syndrome: a 25-year experience. J Pediatr 2004;145:157-63.

28 Pichler J, Horn V, Macdonald S, et al. Intestinal failure-associated liver disease in hospitalised children. Arch Dis Child 2012;97:211-4.

29 Kelly DA. Liver complications of pediatric parenteral nutrition-epidemiology. Nutrition 1998; 14:153-7.

30 Beath S, Pironi L, Gabe S, et al. Collaborative strategies to reduce mortality and morbidity in patients with chronic intestinal failure including those who are referred for small bowel transplantation. Transplantation 2008;85:1378-84.

31 Hughes A, Evans V, Forbes-Penfold D, et al. Burden of care at night when living with a child on parenteral nutrition at home. Clin Nutr ESPEN 2015;10:e180.

32 Dore M, Junco PT, Moreno AA, et al. Ultrashort Bowel Syndrome Outcome in Children Treated in a Multidisciplinary Intestinal Rehabilitation Unit. Eur J Pediatr Surg 2017;27:116-20. 